\title{
Supermode analysis of phase-locked arrays of semiconductor lasers
}

\author{
E. Kapon \\ California Institute of Technology, Pasadena, California 91125 \\ J. Katz \\ Jet Propulsion Laboratory, 4800 Oak Grove Drive, Pasadena, California 91109
}

A. Yariv

California Institute of Technology, Pasadena, California 91125

Received December 23, 1983; accepted February 3, 1984

\begin{abstract}
The optical characteristics of phase-locked semiconductor laser arrays are formulated in terms of the array supermodes, which are the eigenmodes of the composite-array waveguide, by using coupled-mode theory. These supermodes are employed to calculate the near fields, the far fields, and the difference in the longitudinal-mode oscillation wavelengths of the array. It is shown that the broadening in the far-field beam divergence, as well as the broadening of each of the longitudinal modes that were observed in phase-locked arrays, may arise from the excitation of an increasing number of supermodes at increasing pumping levels.
\end{abstract}

Phase locking of several diode lasers that are integrated in parallel provides a useful means for obtaining high-power injection lasers having low beam divergence. ${ }^{1-7}$ Moreover, it was recently demonstrated that phase-locked arrays incorporating separate laser contacts $^{7}$ also exhibit a remarkable degree of longitudinal-mode selectivity as well as output-wavelength tunability. ${ }^{8}$ Many of the observed characteristics of these useful devices, however, are yet not fully understood. The only attempts to explain the optical properties of phase-locked arrays have been limited, to date, to evaluating the array far field, assuming that it consists of identical radiators, ${ }^{2}$ and to deriving the phase relationship between adjacent emitters. ${ }^{9}$

In this Letter, we present an optical model of phaselocked semiconductor laser arrays. This model yields the optical characteristics of the array in terms of its supermodes, i.e., the eigenmodes of the composite-array waveguide. For some special, yet important, cases we calculate analytically the near fields, the far fields, and the propagation constants of these supermodes.

Consider an array of $N$ coupled lasers, as shown in Fig. 1. Each individual laser waveguide, when isolated from its neighbors, is presumed to support a single, TE-like, spatial mode. This mode is described by its electric field $\mathscr{G}_{l}(x, y) \exp \left(i \hat{\beta}_{l} z\right), l=1,2, \ldots N$, where $\hat{\beta}_{l}$ is the complex propagation constant. The total electric field of the array is

$$
E_{y}(x, y, z)=\sum_{l=1}^{N} \mathscr{E}_{l}(x, y) A_{l}(z) \exp \left(i \hat{\beta}_{l} z\right),
$$

where the $z$ dependence of $A_{l}(z)$ is due to the interaction among the array elements. Assuming only the nearest-neighbor coupling, the coupled-mode equations ${ }^{10}$ for the $N$-channel array can be written in the form

$$
\mathrm{d} \mathbf{E} / \mathrm{d} z=i \tilde{M} \mathbf{E},
$$

where $\mathbf{E}$ is a vector whose elements are $E_{l} \equiv A_{l} \exp \left(i \hat{\beta}_{l} z\right)$ and the only nonvanishing elements of the matrix $\bar{M}$ are $\mathcal{M}_{l, l}=\hat{\beta}_{l}$, with $l=1,2, \ldots N$, and $\mathcal{M}_{l, l+1}=\kappa_{l, l+1}, M_{l+1, l}$ $=\kappa_{l+1, l}$, with $l=1,2, \ldots N-1$. The definition of the coupling coefficients $\kappa_{i j}$ is the same as in the case of a pair of coupled waveguides. ${ }^{11}$

The array supermodes are, by definition, the eigensolutions of Eq. (2), i.e., those vectors that satisfy

$$
\mathbf{E}^{\nu}(z)=\mathbf{E}^{\nu}(0) \exp \left(i \sigma_{\nu} z\right)
$$

$\sigma_{\nu}$ being the propagation constant of the supermode $\mathbf{E}^{\nu}$. Substitution of Eq. (3) into Eq. (2) gives

$$
\left(\tilde{\mathcal{M}}-\sigma_{\nu} \tilde{I}\right) \mathbf{E}^{\nu}=0
$$

where $\tilde{I}$ is the unit matrix.

A solution of Eq. (4) yields the $N$ supermodes that are supported by an array of $N$ single-mode lasers. The eigenvectors $\mathbf{E}^{\nu}, \nu=1,2, \ldots N$, can be used in Eq. (1) to evaluate the near field of each supermode; each such mode say, $\mathbf{E}^{\nu}$, describes a phase-locked combination of the individual laser modes with amplitudes $E_{l}{ }^{\nu}$. Gen-

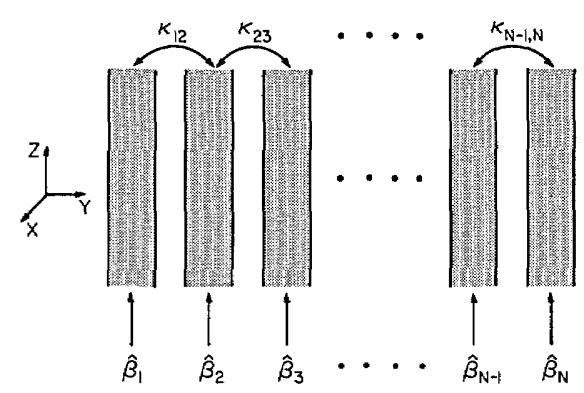

Fig. 1. Schematic illustration of an $N$-channel laser array. The $y$ axis is in the $p-n$-junction plane. 
Table 1. Propagation Constants of the Supermodes of Identical-Channel Arrays

\begin{tabular}{|c|c|}
\hline $\begin{array}{l}\text { Number of } \\
\text { Channels } N\end{array}$ & $\begin{array}{l}\text { Propagation } \\
\text { Constants } \sigma_{\nu}\end{array}$ \\
\hline $\begin{array}{l}2 \\
3 \\
4 \\
5\end{array}$ & 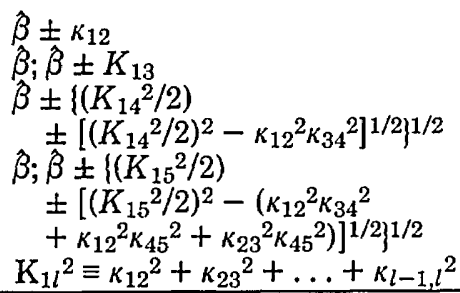 \\
\hline
\end{tabular}
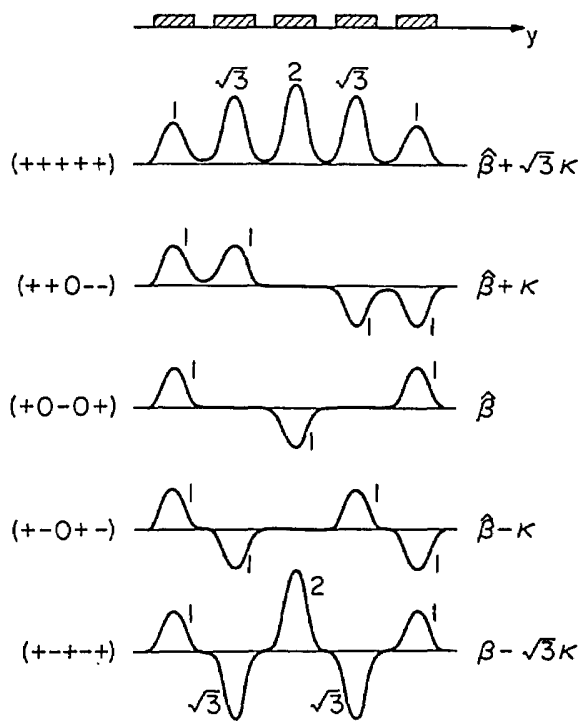

Fig. 2. Schematic illustration of the supermodes in a fivechannel array of equal waveguides $\left(\hat{\beta}_{j}=\hat{\beta}\right)$ and uniform coupling $\left(\kappa_{i j}=\kappa\right)$. The numbers beside the near-field lobes indicate the relative magnitude of the field amplitude. The expressions beside each field pattern are the corresponding supermode eigenvalues.

erally, the arrayinear field will consist of a superposition of the near fields of a number of supermodes. The different propagation constants $\sigma_{\nu}$ of the different supermodes may give rise to a group of Fabry-Perot (FP) resonances associated with a given longitudinal mode of the laser cavity. The wavelength separation $\Delta \lambda_{0} \mathrm{~S}$ between two such modes, which is usually smaller than the FP mode spacing $\Delta \lambda_{0} \mathrm{FP}$, is given by

$$
\Delta \lambda_{0} \mathrm{~S}=(\Delta \beta L / \pi) \Delta \lambda_{0}{ }^{\mathrm{FP}},
$$

where $\Delta \beta$ is the difference in the supermode propagation constants and $L$ is the laser-cavity length.

The supermode near fields can be readily employed to evaluate the far-field radiation pattern of each supermode. In the case of arrays with similar individual near fields $\mathscr{E}_{l}=\mathscr{E}$, the far-field intensity pattern in the junction plane $(y-z$ plane in Fig. 1$)$ is given by

$$
F^{\nu}(\theta)=E(\theta) G^{\nu}(\theta) \text {, }
$$

with

$$
G^{\nu}(\theta) \equiv\left|\sum_{l=1}^{N} \hat{\rho}_{11^{\nu}} \exp \left(i k_{0} S \sin \theta\right)\right|^{2}
$$

and where $E(\theta)$ is the far-field pattern of each individual array element, $\hat{\rho}_{1 l^{\nu}} \equiv E_{l}{ }^{\nu} / \mathrm{E}_{1}{ }^{\nu}$ are the admixture factors, $S$ is the center-to-center separation of adjacent lasers, $\theta$ is the angle in the junction plane, and $k_{0}=2 \pi / \lambda_{0}, \lambda_{0}$ being the free-space wavelength.

Generally, the eigenvalues of a given $N$-channel array can be found by solving Eq. (4) numerically. It is useful, however, to consider special cases that allow for analytic solution and provide some physical insight. The simplest case is that of an array with identical channels, $\hat{\beta}_{1}$ $=\hat{\beta}_{2}=\ldots=\hat{\beta}_{N} \equiv \hat{\beta}$, with uniform coupling, i.e., $\kappa_{i j}=$ $\kappa$. In this case, the solution of Eq. (4) is

$$
\begin{aligned}
E_{l}{ }^{\nu} & =\sin \left(l \frac{\pi \nu}{N+1}\right), \\
\sigma_{\nu} & =\hat{\beta}+2 \kappa \cos \left(\frac{\pi \nu}{N+1}\right), \quad \nu=1,2, \ldots N .
\end{aligned}
$$

Note that the splitting in the propagation constants of the supermodes is proportional to the coupling coefficient $\kappa$. When $N \gg 1$, these propagation constants form a quasi-continuum in the range $\hat{\beta}-2 \kappa \leqslant \sigma \leqslant \hat{\beta}+$ $2 \kappa$, and the maximum wavelength splitting in the FP modes becomes [see Eq. (5)]

$$
\Delta \lambda_{0} \mathrm{~S}=(4 / \pi) \kappa L \Delta \lambda_{0}{ }^{\mathrm{FP}} .
$$

Since usually $\kappa L \lesssim 1$, the excitation of several supermodes would result in an effective broadening of each of the longitudinal modes of the laser array. Such a broadening of the FP modes with increasing pump current was indeed observed experimentally. ${ }^{2}$

In the case of similar channels $\left(\hat{\beta}_{j}=\hat{\beta}\right)$ but nonuni-

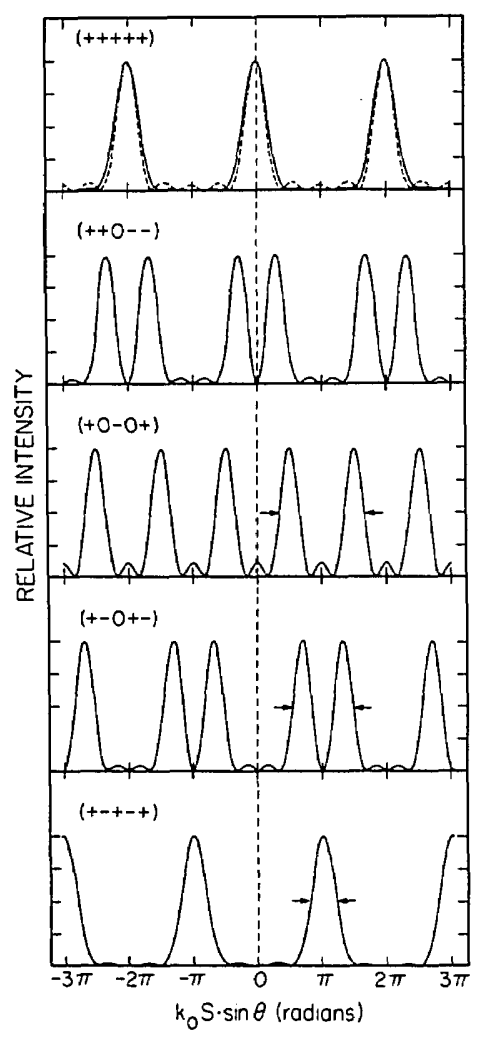

Fig. 3. The grating function $G$ for the supermodes of Fig. 2 . The dashed curve corresponds to an array of five identical radiators. 


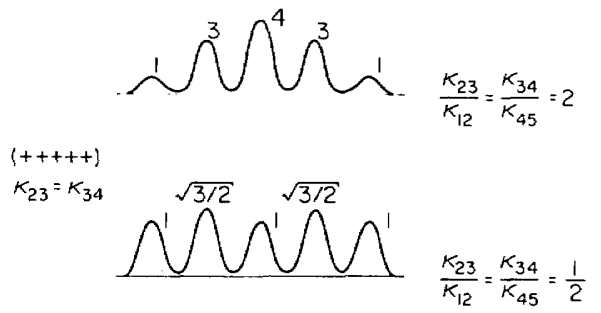

Fig. 4. Effect of variation of the coupling coefficients on the near field of the $(+++++)$ supermode for $\kappa_{23}=\kappa_{34}$.

form coupling $\left(\kappa_{i j} \neq \kappa_{l m}\right.$ for $i j \neq l m$ ), Eq. (4) was solved analytically for $N \leqslant 5$. The eigenvalues for this case are summarized in Table 1 . Note that in this case the admixture factors $\hat{\rho}_{1 l}$ depend only on the ratios of the coupling coefficients of adjacent pairs of channels. In what follows, we illustrate in more detail the supermode features of an array of five identical lasers.

Figure 2 shows schematically the near-field patterns of the supermodes in such an array when the coupling is uniform. Note that the relative excitation of the channels is different in different supermodes. In particular, some of the supermodes are characterized by unexcited channels (which is indicated by a 0 in the notation of Fig. 2). These peculiar forms of the supermode near fields have an important effect on the value of the saturated-gain coefficient in each channel for a given current combination through the array lasers and a given total output intensity. ${ }^{12}$ The modal gain of a given supermode depends on the phase relationship between the fields in adjacent channels, which determines the supermode intensity in the regions between the pumped laser stripes. For example, in the case of more-or-less equal currents, which are injected mainly below each laser stripe contact, it is expected that the $(+-+-+)$ mode would have the lowest threshold since the unpumped regions correspond to a small modal intensity.

Figure 3 shows the grating function $G[\mathrm{Eq}$. (6b)] for the supermodes of Fig. 2. The actual far-field intensity patterns are obtained by superimposing upon these curves the envelope function $E(\theta)$. In practical arrays (e.g., GaAs arrays with $S \approx 10 \mu \mathrm{m}$ and $\sim 4-\mu \mathrm{m}$ laser stripes) one can concentrate on the region $\left|k_{0} S \sin \theta\right|$ $\lesssim 2 \pi$, outside which $E(\theta)$ is practically zero. For comparison, we also show the grating function for (five) identical radiators (dashed curve, Fig. 3), which was used in Ref. 2. Note that the main lobes in the far-field patterns of the supermodes that are characterized by unexcited channels are displaced with respect to those of the $(+++++)$ and $(+-+-+)$ ones. Thus it is clear that, when several supermodes are excited, each will contribute to an effective angular divergence in the far field, as indicated by the arrows in Fig. 3. Thus angular divergencies that can be almost as much as four times the diffraction-limited width are expected. This may explain the wide beam divergencies that are observed with most arrays. $1,3,4,6$ For the GaAs five-element array of Ref. 1 with $\lambda_{0}=0.8 \mu \mathrm{m}$ and $S=8 \mu \mathrm{m}$, we find that the effective angular divergence (FWHP) of the $(+-+-+),(+-0+-)$, and $(+0-0+)$ supermodes is $1.2^{\circ}$, $2.6^{\circ}$, and $3.8^{\circ}$, respectively. The experimental results ${ }^{1}$ show a broadening of the main lobe in the far field from $1.9^{\circ}$ at $I=I_{\text {th }}$ to $3.8^{\circ}$ at $I=2.1 \times I_{\text {th }}$, which was accompanied by the appearance of a structure in this main lobe, in qualitative agreement with our prediction.

Finally, we briefly discuss the effect of varying the coupling coefficients $\kappa_{i j}$. Figure 4 shows the near fields of the $(+++++)$ supermode for $\kappa_{23}=\kappa_{34}$ and for two values of $\kappa_{23} / \kappa_{12}=\kappa_{34} / \kappa_{45}$. Variations in $\kappa_{i j}$ can be accomplished by fabricating the array with different spacing of the laser stripes or by controlling the coupling by using additional contacts intermediate to the laser stripes. ${ }^{13}$ Decreasing the coupling of the two outermost lasers results in further decrease in their excitation in the $(+++++)$ supermode. A stronger coupling of the outermost lasers yields a more uniform excitation in the near field. This illustrates the potential use of the coupling coefficients in tailoring the near fields of a phase-locked array.

In conclusion, we have presented an optical model for phase-locked semiconductor laser arrays that is based on the array supermodes. The description of the array optical field in terms of these supermodes, which are derived by coupled-mode theory, is intermediate between treating the array as a single, giant waveguide and viewing it as a group of coupled waveguides. This model uses the eigenmodes of the total, composite-array waveguide while maintaining and using the information on the coupling between the array elements.

The research described in this Letter was performed jointly by the Applied Physics Department, California Institute of Technology, under contracts with the U.S. Office of Naval Research and the National Science Foundation, and the Jet Propulsion Laboratory under contracts with the National Aeronautics and Space Administration. E. Kapon acknowledges the support of a Weizmann Postdoctoral Fellowship.

\section{References}

1. D. R. Scifres, R. D. Burnham, and W. Streifer, Appl. Phys. Lett. 33, 1015 (1978).

2. D. R. Scifres, W. Streifer, and R. D. Burnham, IEEE J. Quantum Electron. QE-15, 917 (1979).

3. D. R. Scifres, C. Lindstrom, R. D. Burnham, W. Streifer, and T. L. Paoli, Electron. Lett. 19, 169 (1983).

4. D. E. Ackley and R. W. H. Engleman, Appl. Phys. Lett. 39, 27 (1981).

5. W. T. Tsang, R. A. Logan, and R. P. Salathe, Appl. Phys. Lett. 34, 162 (1979).

6. D. Botez and J. C. Connolly, Appl. Phys. Lett. 43, 1096 (1983).

7. J. Katz, E. Kapon, C. Lindsey, S. Margalit, U. Shreter, and A. Yariv, Appl. Phys. Lett. 43, 521 (1983).

8. E. Kapon, J. Katz, S. Margalit, and A. Yariv, Appl. Phys. Lett. 44, 157 (1984).

9. K. Otsuka, Electron. Lett. 19, 723 (1983).

10. A. Yariv, IEEE J. Quantum Electron. QE-9, 919 (1973).

11. A. Yariv, Introduction to Optical Electronics, 2nd ed. (Holt, Rinehart \& Winston, New York, 1976), Chap. 13.

12. J. Katz, E. Kapon, S. Margalit, and A. Yariv (unpublished).

13. E. Kapon, J. Katz, C. Lindsey, S. Margalit, and A. Yariv, Appl. Phys. Lett. 43, 421 (1983). 